\title{
Occupational exposure to magnetic fields and electric shocks and risk of ALS: The Swiss National Cohort
}

\author{
ANKE HUSS ${ }^{1,2}$, ADRIAN SPOERRI ${ }^{2}$, MATTHIAS EGGER ${ }^{2,3}$, HANS KROMHOUT $^{1}$ \& \\ ROEL VERMEULEN ${ }^{1,4}$; FOR THE SWISS NATIONAL COHORT
}

\author{
${ }^{1}$ Institute for Risk Assessment Sciences, Utrecht University, The Netherlands, ${ }^{2}$ Institute of Social and Preventive \\ Medicine (ISPM), University of Bern, Switzerland, ${ }^{3}$ School of Social and Community Medicine, University of \\ Bristol, UK, and ${ }^{4} \mathcal{F u l i u s}$ Centre for Public Health Sciences and Primary Care, University Medical Centre, Utrecht, \\ The Netherlands
}

\begin{abstract}
Amyotrophic lateral sclerosis (ALS) has been associated with exposures in so-called 'electrical occupations'. It is unclear if this possible link may be explained by exposure to extremely low-frequency magnetic fields (ELF-MF) or by electrical shocks. We evaluated ALS mortality in 2000-2008 and exposure to ELF-MF and electrical shocks in the Swiss National Cohort, using job exposure matrices for occupations at censuses 1990 and 2000. We compared 2.2 million workers with high or medium vs. low exposure to ELF-MF and electrical shocks using Cox proportional hazard models. Results showed that mortality from ALS was higher in people who had medium or high ELF-MF exposure in both censuses (HR 1.55 (95\% CI 1.11-2.15)), but closer to unity for electrical shocks (HR 1.17 (95\% CI 0.83-1.65)). When both exposures were included in the same model, the HR for ELF-MF changed little (HR 1.56), but the HR for electric shocks was attenuated to 0.97 . In conclusion, there was an association between exposure to ELF-MF and mortality from ALS among workers with a higher likelihood of long-term exposure.
\end{abstract}

Key words: Amyotrophic lateral sclerosis, occupational exposure, magnetic fields, electric shocks

\section{Introduction}

Amyotrophic lateral sclerosis (ALS) is a progressive neurodegenerative disease with no cure. The large majority of cases are estimated to be sporadic (1), so environmental factors may play a role in the development of the disease. ALS has been associated with exposures in so-called 'electrical occupations'. Studies that assessed risk in workers exposed to extremely low-frequency magnetic fields (ELF-MF) showed mixed results $(2,3)$, and one hypothesis is that electrical shocks are the more relevant exposure. Occupations with elevated ELF-MF exposure are often those in which workers are at higher risk of electrical shocks (4), but the potential risks of these exposures have not been previously disentangled.

Job exposure matrices (JEMs) exist for ELF-MF exposures (5) and have recently been developed for risk of electrical shocks at work $(4,6)$. These JEMs allow researchers to assign exposures for specific occupations. We analysed the Swiss National Cohort (SNC) and evaluated the association between mortality from ALS and ELF-MF exposure, and also with the risk of electrical shocks at work.

\section{Method}

Swiss National Cohort

The SNC is a longitudinal study based on the national censuses of 1990 and 2000, which were linked to death or emigration records by deterministic and probabilistic record linkage methods (7). Linkage was based on key variables of both datasets (gender, date of birth, place of residence, marital status, religion, nationality, date of birth of partner). Standardized reporting of deaths is mandatory in Switzerland, as was participation in the censuses. Coverage of the census 2000 was virtually complete $(98.6 \%)(8)$ and $94 \%$ of deaths could be linked to a 
census record. Because linkage of younger persons is less complete (9), we excluded persons younger than 30 years of age at time of entry (2.59 million) from the analysis. Census information includes information on each individual, comprising educational level, vocational training and occupation. The Cantonal ethics committees of Bern and Zurich approved the Swiss National Cohort. More information on the cohort is available at www.swissnationalcohort.ch.

\section{Outcome and exposure information}

We analysed death from ALS (International Classification of Diseases and Related Health Problems, version 10; code G12.2) registered anywhere on the death certificate. For the exposure assessment, we used two dedicated job exposure matrices: for ELF-MF, we used a modified version of a previously published JEM (5). The JEM assigns exposure as low, medium or high. The median intensity of these magnetic field categories based on the original ELF-MF JEM translated into $0.11 \mu \mathrm{T}$ for low, $0.19 \mu \mathrm{T}$ for medium and $0.52 \mu \mathrm{T}$ for highly exposed, respectively (10). These originally intensity-based-only ratings were up- or down-graded (about $1 \%$ of jobs each), depending on estimated probabilities of exposure (4). The electrical shock JEM defines risk of electrical injury at work. For this JEM, we have previously pooled national accident registry data from five European countries including Switzerland (4). The electrical shock JEM classifies occupational titles according to risk of electric injury as a proxy for occurrence of electric shocks. The rates of pooled electrical injury were categorized at the 75 th and 90 th percentiles into low, medium and high risk. For $67 \%$ of the job codes at the three digit ISCO 88 level $(n=116)$, classification of exposure fell in the same category for ELF-MF and electrical shocks. For the levels high, medium and low the percentages were $2 \%$, $6 \%$ and $59 \%$, respectively.

We also tested whether workers in so-called 'electrical occupations' were at higher risk of ALS, using job titles as previously reported by others $(11,12)$. The respective job titles are provided in Supplementary Table I, which is only available in the online version of the journal. Please find this material with the following direct link to the article: to be found online at http://www.informahealthcare.com/doi/abs/ $10.3109 / 21678421.2014 .896928)$. The combined censuses reported about 18,000 distinct job titles that were already coded to the International Standard Classification of Occupations from 1988 (ISCO88) by the Swiss Federal Office for Statistics. The census also provides information on the (highest) vocational training that people had, and this information is also coded in ISCO88. Since both JEMs are also in ISCO88, exposure could be assigned to the job codes and vocational training codes in the census. Homemakers were set to low exposure, as were people without an occupation at the time of the censuses.

\section{Statistical analysis}

We used age as the underlying time-scale for the Cox proportional hazard models when we analysed the association between high or medium vs. low ELF-MF or shock exposure at work. Exposures were separately evaluated for occupations at each census $(1990,2000)$. We also compared medium or high vs. low exposure over the combined censuses. Pensioners in 1990 were excluded from the analyses that evaluated exposures from 1990, as were pensioners in 2000. All pensioners were excluded from the combined analysis of the censuses 1990 and 2000. We excluded them because the group included retirees and disabled persons who received early pensions. One reason for receiving a disability related pension could be ALS, the outcome of interest evaluated in our study.

Please note that persons registered in the census 1990 but who died before entering the risk set on 5 December 2000 were not included in the data set, resulting in a higher apparent proportion of pensioners in the 2000 census.

We calculated time from date of birth; participants entered the risk set on 5 December 2000, the day of the 2000 census. Observation time ended on the date that came first: 31 December 2008; date of emigration; or death. We tested models for the proportionality assumption using statistical tests based on Schoenfeld residuals, and the assumption was met for all exposure variables. Models were adjusted for gender and nationality (Swiss, other; model I). We found no association between risk of ALS and educational level, civil status, language region, degree of urbanization of the municipality where persons lived and a neighbourhood index of socioeconomic position based on the 50 nearest households (13) and excluded these variables from the analysis. In model II, we also adjusted for the other exposures (ELF-MF or shocks). We performed several sensitivity analyses:

- We restricted the data set to those workers who had reported working full time (1fte) in both censuses and additionally to those who had been exposed to medium or high ELF-MF levels during their vocational training. In line with a previous publication (12) we further stratified by gender and by the median age at entry into the cohort in the year 2000 for deaths from ALS (56 years, for cases with exposure information).

- We also tried to disentangle effects of the two evaluated exposures. We did so by combining medium/high exposure to ELF-MF with medium/high risk of electric shocks; medium/ high exposure to ELF-MF with low risk of 
electric shocks; low exposure to ELF-MF with medium/high risk of electric shocks vs. low ELF-MF exposure and low risk of shocks at both censuses.

- Finally, we adjusted for other occupational exposures (pesticides, herbicides, chlorinated solvents and heavy metals, using the ALOHA-JEM) (14) to determine if that changed our HRs. All analyses were performed using Stata version 12 (StataCorp, College Station, Texas, USA).

\section{Results}

A total of 1358 persons died from ALS; nine people who died were under 30 years of age and were excluded from the analysis; 87 could not be linked to a census record. The 1990 census contained information on 4,240,812 persons (1230 cases); we excluded 659,086 pensioners (411 cases) and 182,913 persons (27 cases) for whom 1990 occupational information was missing. This left us with $3,398,813$ persons (792 cases) in the analysis for 1990. The 2000 census contained information on 4,689,667 persons (1262 cases); we excluded $1,320,102$ pensioners (880 cases) and 816,883 persons (83 cases) for whom 2000 occupation information was missing. This left us with 2,552,682 persons (299 cases) in the analysis for 2000. A total of $2,167,046$ persons (278 cases) contributed data for the analysis of both censuses. Characteristics are given in Table II and in Supplementary Table II, which is only available in the online version of the journal. Please find this material with the following direct link to the article: to be found online at http:// www.informahealthcare.com/doi/abs/10.3109/2167 8421.2014.896928).

When analysed separately, hazard ratios (HR) were around unity for those exposed to ELF-MF, for workers at risk of electrical shocks, or employed in electrical occupations in either 1990 or 2000, without exposure-response relationship with intensity of exposure (Table II). We observed an increase in mortality from ALS for ELF-MF exposures in persons classified as medium/high exposed in both 1990 and 2000; the increase was lower for electrical shocks. After we accounted for both exposures in the same model, we found ELF-MF had maintained almost the same HR, but the HR for electrical shocks was attenuated (Table III); 21 cases were classified as medium/high exposed to ELF-MF and shocks in both censuses. In line with this result, persons who had been exposed to medium or high ELF-MF levels for at least one of the censuses but not to shocks had an increased risk of 1.51 (95\% CI 1.08-2.11) compared to persons with low exposures at both censuses. Persons with medium or high risk of electrical shocks for at least one of the censuses but not to ELF-MF had a HR of $1.12(95 \%$ CI $0.77-1.64)$.

Over both censuses combined, only one person dying from ALS had worked in an electrical occupation (both definitions, data not shown) and only three had been high exposed in both censuses. HRs increased slightly for medium/high ELF-MF vs. low exposure when we reduced the data set to those who had reported working $1 \mathrm{fte}$ in both censuses, or who had also been exposed during their vocational training (Table III).

HRs were not materially different in males and females (interaction $p$-value $=0.9$ ), and did not differ by median age at entry (interaction $p$-value $=0.4$ ). Adjusting for potential other occupational exposures (pesticides, herbicides, chlorinated solvents and heavy metals) had no material effect on HRs (data not shown).

\section{Discussion}

In a large database covering the Swiss population, we observed no increased risks of ALS in persons with elevated ELF-MF exposure, higher risk of electrical shock or in workers with an electrical occupation when exposures were based on census data from either 1990 or 2000. People who were occupationally exposed to elevated ELF-MF at both censuses had an elevated risk of ALS (HR of 1.55; 95\% CI 1.11-2.15). No elevated risk of ALS was observed for persons at risk of electrical shocks at the timepoints of both censuses. Our study thus does not support the hypothesis that shocks may be the more relevant exposure in the generation of ALS.

Some concerns remain regarding under-ascertainment of ALS when using death certificates (15). The crude mortality rate in our population, however,

Table I. Study characteristics, combined information from census 1990 and 2000.

\begin{tabular}{|c|c|c|c|c|c|c|}
\hline & $n$ & $n$ cases & $\%$ females & $\begin{array}{c}\text { median age } \\
\text { at entry (yrs) }\end{array}$ & $\begin{array}{l}\% \text { foreign } \\
\text { nationals }\end{array}$ & $\begin{array}{c}\% \text { working } \\
1 \mathrm{fte}\end{array}$ \\
\hline \multicolumn{7}{|l|}{ ELF-MF JEM } \\
\hline Low in 1990 and 2000 & $1,572,241$ & 191 & 54 & 46 & 11 & 43 \\
\hline Medium/high in 1990 or 2000 & 354,223 & 41 & 41 & 44 & 21 & 50 \\
\hline Medium/high in 1990 and 2000 & 240,582 & 46 & 22 & 46 & 21 & 76 \\
\hline \multicolumn{7}{|l|}{ Electrical shocks JEM } \\
\hline Low in 1990 and 2000 & $1,556,258$ & 200 & 59 & 46 & 10 & 40 \\
\hline Medium/high in 1990 or 2000 & 313,273 & 34 & 30 & 43 & 23 & 55 \\
\hline Medium/high in 1990 and 2000 & 297,515 & 44 & 9 & 44 & 22 & 84 \\
\hline
\end{tabular}


Table II. Risk of death from ALS (2000-2008) and exposure to ELF-MF, electrical shocks at work or working in an electrical occupation in either 1990 or 2000.

\begin{tabular}{|c|c|c|c|c|}
\hline & \multicolumn{2}{|c|}{ Census 1990} & \multicolumn{2}{|c|}{ Census 2000} \\
\hline & $n$ cases & $\mathrm{HR}(95 \% \mathrm{CI})$ & $n$ cases & $\operatorname{HR}(95 \% \mathrm{CI})$ \\
\hline \multicolumn{5}{|c|}{ ELF-MF JEM } \\
\hline Low & 606 & referent & 240 & referent \\
\hline Medium & 163 & $1.21(1.03-1.46)$ & 55 & $1.20(0.90-1.62)$ \\
\hline High & 23 & $1.11(0.73-1.69)$ & 4 & $0.64(0.24-1.73)$ \\
\hline \multicolumn{5}{|c|}{ Electrical shocks JEM } \\
\hline Low & 625 & referent & 238 & referent \\
\hline Medium & 102 & $0.90(0.72-1.11)$ & 41 & $1.13(0.81-1.57)$ \\
\hline High & 65 & $1.19(0.92-1.55)$ & 20 & $1.11(0.70-1.76)$ \\
\hline \multicolumn{5}{|c|}{ Electrical occupation (Deapen, 1986) } \\
\hline No & 774 & referent & 297 & referent \\
\hline Yes & 18 & $1.20(0.75-1.93)$ & 2 & $0.36(0.09-1.45)$ \\
\hline \multicolumn{5}{|c|}{ Electrical occupation (Feychting 2003) } \\
\hline No & 782 & referent & 297 & referent \\
\hline Yes & 10 & $1.10(0.59-2.05)$ & 2 & $0.64(0.16-2.57)$ \\
\hline
\end{tabular}

For the analysis of ALS risk in electrical occupations, job titles used in two previous publications were applied $(11,12)$. Analyses were adjusted for gender and nationality, age was used as underlying time scale.

was 2.26 per 100,000 person-years, which is well in line with incidence data that have been reported from other European countries (16). In addition, it is unlikely that such under-ascertainment would be related to the evaluated exposures, which in turn would mean that using death certificates would not be expected to cause biased results, but loss of power and thus a decreased ability to detect any association. Our analysis offers nearly complete coverage of the Swiss population; occupational information was prospectively collected. Exposure information was only available at the time the censuses were taken, so that we could not analyse cumulative exposure, or the timing of exposure over a lifetime. We could however, determine if persons had been exposed at one or two censuses and additionally during vocational training, and we found that risk increased for those exposed at least at two time-points (indicating a longer exposure duration).

We observed no increased risks for persons in occupations at higher risk of electrical shocks, but the power of our study to exclude such an association was limited. Our shock-JEM is based on accident data about electrical injuries and may not have captured other electrical exposures at work, such as electric fields, contact currents or nuisance shocks,

Table III. Risk of death from ALS (2000-2008) and exposure to ELF-MF, electrical shocks in 1990 and 2000 .

\begin{tabular}{|c|c|c|c|}
\hline Census 1990 and 2000 & $n$ cases & $\begin{array}{c}\text { Model I } \\
\text { HR }(95 \% \text { CI) }\end{array}$ & $\begin{array}{c}\text { Model II } \\
\text { HR }(95 \% \text { CI })\end{array}$ \\
\hline \multicolumn{4}{|l|}{ ELF-MF } \\
\hline Low in 1990 and 2000 & 191 & referent & referent \\
\hline Medium/high in 1990 or 2000 & 41 & $1.09(0.77-1.53)$ & $1.12(0.79-1.61)$ \\
\hline Medium/high in 1990 and 2000 & 46 & $1.55(1.11-2.15)$ & $1.56(1.09-2.25)$ \\
\hline Medium/high in 1990 and $2000^{a}$ & 30 & $1.59(1.07-2.35)$ & $1.30(0.77-2.19)$ \\
\hline Medium/high in 1990 and $2000^{\mathrm{b}}$ & 40 & $1.66(1.15-2.42)$ & $1.68(1.11-2.55)$ \\
\hline Medium/high in 1990 and $2000^{c}$ & 28 & $1.71(1.11-2.61)$ & $1.42(0.81-2.48)$ \\
\hline \multicolumn{4}{|l|}{ Electrical shocks } \\
\hline Low in 1990 and 2000 & 200 & referent & referent \\
\hline Medium/high in 1990 or 2000 & 34 & $0.94(0.65-1.36)$ & $0.90(0.61-1.33)$ \\
\hline Medium/high in 1990 and 2000 & 44 & $1.17(0.83-1.65)$ & $0.97(0.66-1.42)$ \\
\hline Medium/high in 1990 and $2000^{\mathrm{d}}$ & 31 & $1.13(0.76-1.69)$ & $1.20(0.73-1.98)$ \\
\hline Medium/high in 1990 and $2000^{\mathrm{e}}$ & 40 & $1.20(0.82-1.76)$ & $0.97(0.63-1.49)$ \\
\hline Medium/high in 1990 and $2000^{\mathrm{f}}$ & 29 & $1.21(0.79-1.86)$ & $1.26(0.73-2.14)$ \\
\hline
\end{tabular}

Model I: using age as the underlying time scale, and adjusted for gender and nationality. Model II: additionally adjusted for the respective other exposure. ${ }^{a}$ additionally medium/high ELF-MF exposed during vocational training; bersons reporting to work $1 \mathrm{fte}$ in both censuses; "both a and b;

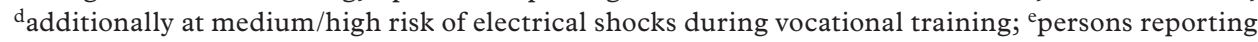
to work $1 \mathrm{fte}$ in both censuses; ${ }^{\mathrm{f}}$ both $\mathrm{d}$ and e. Note that HRs of a-f are based on smaller data sets and therefore do not compare to the identical referent group as in the other analyses shown in this table. 
which might more commonly occur in occupations classified as 'electrical' (17). However, we observed no increased risks in workers in 'electrical occupations'. This is in contrast to earlier publications that reported more consistently higher risks in electrical occupations $(11,12,18,19)$ than in studies evaluating ELF-MF exposures: of studies addressing ELF-MF exposures, some reported positive associations (20-23) while others did not $(12,19,24)$. Interestingly, of the studies that report effects, most are cohort studies performed in industrial cohorts with often more complete job history information. The publications that found no increased risks of ALS from the exposure to magnetic fi elds, in contrast, are more often general population studies with occupations only reported at one singe time-point, such as from death certificates (25-27) or from a baseline questionnaire (24). Electrical shocks were previously analysed from self-report, with inconsistent results $(11,28)$. One recent Danish study addressed risks in workers who had survived electric injury, but was underpowered to detect potential associations with ALS (29).

In summary, our study provided no evidence that ALS is associated with electrical shocks at work. We did find that ALS is associated with occupational exposure to medium or high levels of extremely lowfrequency magnetic fi elds among workers with a higher likelihood of being long-term exposed to ELF-MF.

\section{Acknowledgements}

We would like to thank Judith Lupatsch for help with inspection of the ISCO88 codes, and Joseph Bowman for making the original ELF-MF JEM available, and the Swiss Federal Statistical Office, whose support made the Swiss National Cohort possible. We also thank Kali Tal for her editorial assistance.

Declaration of interest: All authors declare that they have no confl icts of interest. The authors alone are responsible for the content and writing of the paper.

This study was supported by ZonMW within the programme Electromagnetic Fields and Health Research, The Netherlands (grant No. 85800001). The Swiss National Cohort is supported by the Swiss National Science Foundation (grant No. 33CS30_134273/1).

\section{References}

1. Cannon JR, Greenamyre JT. The role of environmental exposures in neurodegeneration and neurodegenerative diseases. Toxicological Sciences. 2011;124:225-50.

2. Kheifets L, Bowman JD, Checkoway H, Feychting M, Harrington J, Kavet R, et al. Future needs of occupational epidemiology of extremely low frequency electric and magnetic fields: review and recommendations. Occup Environ Med. 2009;66:72-80.
3. Zhou H, Chen G, Chen C, Yu Y, Xu Z. Association between extremely low-frequency electromagnetic fields occupations and amyotrophic lateral sclerosis: a meta-analysis. PloS One. 2012;7:e48354.

4. Huss A, Vermeulen R, Bowman JD, Kheifets L, Kromhout H. Electric shocks at work in Europe: development of a job exposure matrix. Occup Environ Med. 2013;70:261-7.

5. Bowman JD, Touchstone JA, Yost MG. A population based job exposure matrix for power-frequency magnetic fields. J Occup Environ Hyg. 2007;4:715-28.

6. Vergara XP, Kheifets L, Silva M, Bracken TD, Yost M. New electric shock job exposure matrix. Am J Ind Med. 2012;55:232-40.

7. Spoerri A, Zwahlen M, Egger M, Bopp M. The Swiss National Cohort: a unique database for national and international researchers. International Journal of Public Health. 2010;55:239-42.

8. Renaud A. Methodology report: coverage estimation for the Swiss population census 2000. Neuchâtel, Switzerland: Swiss Federal Statistical Office. 2004.

9. Bopp M, Spoerri A, Zwahlen M, Gutzwiller F, Paccaud F, Braun-Fahrländer C, et al. Cohort profile: The Swiss National Cohort: a longitudinal study of 6.8 million people. Int J Epidemiol. 2009;38:379-84.

10. Koeman T, Slottje $P$, Kromhout H, Schouten LJ, Goldbohm RA, van den Brandt, et al. Occupational exposure to extremely low-frequency magnetic fields and cardiovascular disease mortality in a prospective cohort study. Occup Environ Med. 2013;70:402-7.

11. Deapen DM, Henderson BE. A case-control study of amyotrophic lateral sclerosis. Am J Epidemiol. 1986;123: 790-9.

12. Feychting M, Jonsson F, Pedersen NL, Ahlbom A. Occupational magnetic field exposure and neurodegenerative disease. Epidemiology. 2003;14:413-9.

13. Panczak R, Galobardes B, Voorpostel M, Spoerri A, Zwahlen M, Egger M. A Swiss neighbourhood index of socioeconomic position: development and association with mortality. J Epidemiol Community Health. 2012; 66:1129-36.

14. Matheson M, Benke G, Raven J, Sim M, Kromhout H, Vermeulen R, et al. Biological dust exposure in the workplace is a risk factor for chronic obstructive pulmonary disease. Thorax. 2005;60:645-51.

15. Chiò A, Magnani C, Oddenino E, Tolardo G, Schiffer D. Accuracy of death certificate diagnosis of amyotrophic lateral sclerosis. J Epidemiol Community Health. 1992;46: 517-8.

16. Chio A, Logroscino G, Traynor BJ, Collins J, Simeone JC, Goldstein LA, et al. Global epidemiology of amyotrophic lateral sclerosis: a systematic review of the published literature. Neuroepidemiology. 2013;41:118-30.

17. Bracken TD, Kavet R, Patterson RM, Fordyce TA. An integrated job exposure matrix for electrical exposures of utility workers. J Occup Environ Hyg. 2009;6:499-509.

18. Graves AB, Rosner D, Echeverria D, Yost M, Larson EB. Occupational exposure to electromagnetic fields and Alzheimer's disease. Alzheimer Dis Assoc Disord. 1999;13:165.

19. Savitz DA, Checkoway H, Loomis DP. Magnetic field exposure and neurodegenerative disease mortality among electric utility workers. Epidemiology. 1998;9:398-404.

20. Håkansson N, Gustavsson P, Johansen C, Floderus B. Neurodegenerative diseases in welders and other workers exposed to high levels of magnetic fields. Epidemiology. 2003;14:420-6.

21. Johansen $\mathrm{C}$, Olsen JH. Mortality from amyotrophic lateral sclerosis, other chronic disorders, and electric shocks among utility workers. Am J Epidemiol. 1998;148:362-8.

22. Röösli M, Lörtscher M, Egger M, Pfluger D, Schreier N, Lörtscher E, et al. Mortality from neurodegenerative disease and exposure to extremely low-frequency magnetic fields: 31 
years of observations on Swiss railway employees. Neuroepidemiology. 2007;28:197-206.

23. Sorahan T, Kheifets L. Mortality from Alzheimer's, motor neuron and Parkinson's disease in relation to magnetic field exposure: findings from the study of UK electricity generation and transmission workers, 1973-2004. Occup Environ Med. 2007;64:820-6.

24. Parlett LE, Bowman JD, van Wijngaarden E. Evaluation of occupational exposure to magnetic fields and motor neuron disease mortality in a population based cohort. Journal of Occupational and Environmental Medicine. 2011;53: 1447-51.

25. Buckley J, Warlow C, Smith P, Hilton-Jones D, Irvine S, Tew J. Motor neuron disease in England and Wales, 19591979. Journal of Neurology, Neurosurgery \& Psychiatry. 1983;46:197-205.
26. Noonan CW, Reif JS, Yost M, Touchstone J. Occupational exposure to magnetic fields in case-referent studies of neurodegenerative diseases. Scand J Work Environ Health. $2002 ; 28: 42-8$.

27. Park RM, Schulte PA, Bowman JD, Walker JT, Bondy SC, Yost MG, et al. Potential occupational risks for neurodegenerative diseases. Am J Ind Med. 2005;48: 63-77.

28. Gunnarsson LG, Bodin L, Söderfeldt B, Axelson O. A case-control study of motor neuron disease: its relation to heritability, and occupational exposures, particularly to solvents. Br J Ind Med. 1992;49:791-8.

29. Grell K, Meersohn A, Schüz J, Johansen C. Risk of neurological diseases among survivors of electric shocks: a nationwide cohort study. Denmark, 1968-2008. Bioelectromagnetics. 2012;33:459-65. 